\title{
The miR-187 Induced Bone Reconstruction and Healing in a Mouse Model of Osteoporosis, and Accelerated Osteoblastic Differentiation of Human Multipotent Stromal Cells by Targeting BARX2
}

\section{Jun Zhang}

Institute of Orthopedic Diseases, The First Affiliated Hospital of Jinan University; Guizhou Orthopedics Hospital

\section{Feng Pan}

Guizhou Orthopedics Hospital

\section{Benseng Tang}

Guizhou Orthopedics Hospital

Jing Li

Guizhou Orthopedics Hospital

Zhengang Zha ( $\sim$ ZhengangZha@163.com)

Institu of Orthopedic Diseases, The First Affiliated Hospital of Jinan Unversity

\section{Research article}

Keywords: osteoporosis, fracture, human marrow-derived mesenchymal stem cells, miR-187, BARX2, osteogenic differentiation

Posted Date: August 26th, 2020

DOI: https://doi.org/10.21203/rs.3.rs-47428/v1

License: (1) This work is licensed under a Creative Commons Attribution 4.0 International License. Read Full License

Version of Record: A version of this preprint was published at Pathology - Research and Practice on March 1st, 2021. See the published version at https://doi.org/10.1016/j.prp.2021.153340. 


\section{Abstract}

Background: Multiple microRNAs (miRNAs) have been proven to regulate osteogenic differentiation by affecting the Runx2 signaling pathway. The intervention of miRNA can delay the progress of osteoporosis (OP) and induce fracture repair by affecting bone regeneration. However, the function and mechanism of miR-187 in osteoporotic fractures are still unknown.

Methods: We first established the OP mouse model. Next, the BMD value was certified by iDXA. The miR187 level in the OP mice and serum of OP patients was identified through qRT-PCR. Bone repair and bone healing were assessed through toluidine blue staining and X-ray, and BARX2 expression was also confirmed. Osteogenesis-related proteins, ALP activity, and the matrix mineralization state were evaluated by western blot, ALP staining, and Alizarin Red staining in hMSCs after transfection with miR-187 mimics, miR-187 inhibitor, or human BarH-like homeobox 2 (BARX2) siRNA. Moreover, the interplay between miR187 and BARX2 was identified through the dual-luciferase reporter.

Results: The BMD value was notably reduced in the OP mice, and miR-187 was markedly downregulated in the OP mice and serum of OP patients. Meanwhile, we proved that miR-187 induced bone reconstruction and healing, and downregulated BARX2 in the OP mouse model. We also proved that BARX2 was a direct target of miR-187, and could be significantly downregulated by miR-187. Furthermore, miR-187 induced osteogenic differentiation of hMSCs by targeting BARX2.

Conclusions: The miR-187 might have a significant therapeutic effect in osteoporotic fractures. miR-187 accelerated osteogenic differentiation of hMSCs by directly regulating BARX2.

\section{Background}

Osteoporosis (OP) is a universal metabolic bone disease and has been recognized as a major public health problem worldwide [1]. Postmenopausal osteoporosis is one of the most widespread types of osteoporosis [2]. Osteoporosis is characterized by reduced bone mass, decreased bone mineral density, and destruction of bone microstructure, which can result in increased bone fragility, and even an increased risk of fracture $[3,4]$. Currently, osteoporotic fractures have become the most serious complication of osteoporosis [5]. Studies have testified that the mortality rate associated with diseases caused by osteoporotic fractures have exceeded the combined death rates of the three major gynecological tumors (breast cancer, cervical cancer, and uterine body cancer), and the related death rates have been increasing year by year $[6,7]$. According to statistics, the occurrence risk of osteoporotic fracture is very high, being about $40-50 \%$ for females and $13-22 \%$ for males $[8,9]$. Currently, OP therapy drugs are broadly divided into basic drugs and anti-absorption drugs. These drugs cannot induce bone formation and have considerable side effects $[10,11]$. Therefore, further studies on the molecular mechanisms of osteoporosis are crucial for therapies for osteoporosis.

The growth of bone is manifested mainly by the enhancement of osteogenic differentiation and the weakening of osteoclast differentiation [12]. Osteoporosis is caused mainly by a decrease in osteogenic 
differentiation and an increase in osteoclast differentiation [13]. Human marrow-derived mesenchymal stem cells (hMSCs) have the potential to differentiate into a variety of cell types. They can also differentiate into osteoblasts and adipocytes under natural conditions, both of which maintain a dynamic balance $[14,15]$. Research has also shown that a disruption in this balance can generate metabolic bone diseases, such as osteoporosis $[10,15]$. An increasing amount of research has also confirmed that boneforming drugs will have a broad application prospect in OP $[16,17]$. Therefore, exploring the mechanism of osteogenic differentiation may provide a new strategy for the treatment of OP.

MicroRNAs (miRNAs) are a class of non-coding single-stranded RNA with a length of 22 nucleotides encoded by endogenous genes $[18,19]$. The miRNAs can mediate specific gene silencing, cause inhibition and degradation of target gene mRNAs, and, thus, regulate protein synthesis [20]. Recent research has testified that miRNAs can be involved in the development process of osteoporosis by regulating the balance between osteogenesis and osteoclasts [21, 22]. Among them, miR-187 has been shown to significantly contribute to a wide range of diseases, such as glaucoma [23], cancer [24-27], and psoriasis [28], etc. In accordance with the literature, miR-187 was highly expressed in the non-fracture group versus fracture group [29]. However, the function and mechanism of miR-187 in OP has not been explored.

In our study, we further investigated the expression of miR-187 in the OP mouse model and the serum of osteoporosis patients. We determined the influence of miR-187 on bone repair and bone healing in the OP mouse model, and also on the osteogenic differentiation of hMSCs. We verified the interplay between miR-187 and human BarH-like homeobox 2 (BARX2) in OP.

\section{Methods}

\section{Animal}

Forty female specific pathogens free (SPF) C57BL/6J mice were supplied by the Animal Experiment Center of the Institute of Radiation Medicine of the Chinese Academy of Medical Sciences. The animals were kept in a room with a room temperature of $15-28^{\circ} \mathrm{C}$, humidity of $45-55 \%$, and a 12 -hour light/dark cycle. Mice had free access to drinking water and standard food. This study obtained the approval of the Animal Experiment Center of the Institute of Radiation Medicine of the Chinese Academy of Medical Sciences animal experiment ethics qualification.

\section{Establishment of the OP model}

After a week of routine feeding, all C57BL/6J mice were anesthetized through intraperitoneal injection of $3 \%$ pentobarbital sodium $(30 \mathrm{mg} / \mathrm{kg}$ ). After skin disinfection, the abdominal midline of the mice was cut about $1.5 \mathrm{~cm}$, and bilateral fallopian tubes and ovaries were exposed. After ligation of the fallopian tubes, the ovaries and the ligated fallopian tubes were removed, and the wound was closed. Penicillin was injected into the abdominal cavity for 3 days. In the sham group, we removed only the same amount of adipose tissue around bilateral ovaries. After surgery, the resected ovarian tissues were collected, and part of the tissues was embedded and sectioned. 


\section{Establishment of the femoral fracture model}

After 4 weeks of regular feeding, the mice in the sham-operated group and OP model group were anesthetized using the same procedure. The femurs of the mice were exposed, a steel needle with a diameter of $0.45 \mathrm{~mm}$ was inserted retrograde from the knee joint for intramedullary fixation. The fracture was formed by cutting off $1 / 3$ of the femur with a scalpel. After disinfection, healing of the wound was regularly observed. Negative control (NC) and miR-187 lentivirus were obtained from the Wuhan Hualian Biotechnology Co. Ltd (Wuhan, China), and the OP mice were injected with NC or miR-187 lentivirus through the tail vein. At the end of the experiment, rats were sacrificed under anesthesia using $50 \mathrm{mg} / \mathrm{kg}$ sodium pentobarbital by intraperitoneal injection.

\section{Clinical samples}

The blood samples of 33 healthy controls and 33 OP patients and the bone marrow samples of OP patients were collected from the First Affiliated Hospital of Jinan University. The serum was isolated from the blood samples through centrifugation $\left(3,000 \times g\right.$ for 5 min at $\left.4{ }^{\circ} \mathrm{C}\right)$. Before collection, informed consent was provided from all patients. This study was allowed by the ethics committee of the The First Affiliated Hospital of Jinan University.

\section{Isolation of hMSCs}

We harvested the hMSCs from the bone marrow samples of OP patients. After washing, the extracted hMSCs were cultured in a-modified Eagle's medium (a-MEM, Hyclone, cat. no. SH30265.01B) containing $2 \%$ glutamine and $10 \%$ fetal bovine serum (FBS, Gibco, Rockville, MD, USA). HEK293 cells were acquired from ATCC and grown in EMDM medium (Hyclone, Cat. no.SH30022.01B) containing 10\% FBS. All cells were incubated at $37^{\circ} \mathrm{C}$ in a $5 \% \mathrm{CO}_{2}$ incubator.

\section{Osteogenic differentiation of hMSCs}

Osteogenic differentiation of hMSCs was induced in the DMEM medium supplemented with $1 \% \mathrm{~L}$ glucose, $10 \%$ FBS, $10 \mathrm{mM}$ b-glycerophosphate, $0.25 \mathrm{mM}$ ascorbic acid, and $10 \mathrm{nM}$ dexamethasone at $37^{\circ} \mathrm{C}, 5 \% \mathrm{CO}_{2}$.

\section{Cell transfection}

The miR-187 mimics, miR-187 inhibitor, BARX2 siRNA, and respective NC were purchased from GenePharma (Shanghai, China). hMSCs $\left(1 \times 10^{5}\right.$ cells/well) were evenly spread in 6-well plates and cultured for $8 \mathrm{~h}$ at $37^{\circ} \mathrm{C}$ before transfecting with miR-187 mimics, miR-187 inhibitor, BARX2 siRNA, or corresponding NC through the use of Lipofectamine 3000 reagent (Invitrogen) for $48 \mathrm{~h}$.

\section{X-ray}

Specimens of the fractured tibiae were collected and immediately photographed using the RADspeed digital X-ray photography system ( $40 \mathrm{kV}, 1,2 \mathrm{~mA})$ to assess bone healing.

\section{Detection of bone mineral density (BMD)}


The iDXA (GE Healthcare Lunar, USA) was used to examine the BMD of the fractured tibiae.

\section{ALP staining}

In line with the instructions of the ALP staining kit (Beyotime, Shanghai, China; cat. no. P0321), the transfected hMSCs were washed using PBS and then fixed for 30 min in 4\% paraformaldehyde. After washing the hMSCs, the proper amount of dye solution was added. After washing, we observed the results under a microscope.

\section{Alizarin Red staining}

After washing the fixed hMSCs in each group, alizarin red S solution (Waldeck GmbH) was added at $37^{\circ} \mathrm{C}$ for $30 \mathrm{~min}$. The dye results were observed using a microscope.

\section{Quantitative real-time PCR (qRT-PCR) assay}

Total RNA was isolated by applying TRIzol reagent (Invitrogen, Carlsbad, CA, USA), and total RNAs were reverted to cDNAs following the instructions of the reverse transcription kit (Invitrogen). Based on the instructions of the SYBR Green PCR kit (Invitrogen), gene expression was examined on the ABI PRISM® 7500 System (Applied Biosystems, Foster City, CA, USA). The relative expression of the target genes was analyzed by $2^{-\triangle \triangle C T}$.

\section{Western blot assay}

Total protein was separated through the application of RIPA buffer (Beyotime) containing 1\% proteinase inhibitor. The BCA method was utilized to determine the protein concentration in each group. Then, $40 \mu \mathrm{g}$ total protein from each group was isolated using 10\% SDS-PAGE under $120 \mathrm{~V}$ constant voltage. The protein was transferred to a PVDF membrane (Roche, Santa Clara, CA, USA; cat. no. 3010040001) under the conditions of $200 \mathrm{~mA}$ for $90 \mathrm{~min}$. After sealing, the membranes were incubated overnight at $4{ }^{\circ} \mathrm{C}$ with the corresponding primary antibodies, which were from Abcam (Burlingame, CA, USA). Then, the membranes were exposed to the secondary antibody for $1 \mathrm{~h}$. The specific proteins were developed using ECL chemiluminescence (KALANG; cat. no. KL-D3490).

\section{Luciferase reporter gene assay}

WT-pmirGLOهBARX2 and Mut-pmirGLOهBARX2 were obtained from Wuhan Hualian Biotechnology Co. Ltd (Wuhan, China). HEK293 cells $\left(1 \times 10^{5}\right.$ cells/well) were co-transfected with the obtained plasmids or miR187 mimics using Lipofectamine 3000 reagent (Invitrogen) for 48 h. The Dual-Luciferase Reporter Assay System (Promega, San Luis Obispo, CA, USA) was used to develop the results.

\section{Statistical analysis}

All experiments were repeated at least three times, and the data are displayed as the mean \pm SD. The data were counted via GraphPad Prism 5.0 software (GraphPad, San Diego, CA, USA) using Student's $t$-test or one-way ANOVA. $P<0.05$ denotes statistical significance. 


\section{Results}

The miR-187 was significantly downregulated in OP.

To determine the change in expression of miR-187 in OP, we first performed a bilateral ovariectomy to establish the OP mouse model. First, we uncovered that the BMD value was observably reduced in the left femur of the mice in the OP model group compared to the sham group $(P<0.001$, Fig. $1 \mathrm{~A})$. Second, our data proved that miR-187 expression was lower in the femoral bone of the OP mice than in the sham mice $(P<0.01$, Fig. 1B). The level of miR-187 was markedly downregulated in the serum of OP patients ( $\mathrm{n}$ $=33)$ relative to the healthy controls $(n=33)(P<0.001$, Fig. $1 C)$. These results proved that the expression of miR-187 in OP was low.

The miR-187 markedly promoted the reconstruction and healing of bone and downregulated BARX2 in the OP mouse model.

To further study whether miR-187 has a significant effect during OP pathogenesis, we injected OP mice with miR-187 or NC lentivirus through the tail vein. The data from the qRT-PCR analysis exhibited a significant decline in the expression of miR-187 in the femoral bone of the OP mice versus the sham mice. This decline could be partially reversed by miR-187 overexpression in the femoral bone of the OP mice $(P<0.05$, Fig. 2A). In addition, our results of toluidine blue staining disclosed that the relative bone repair rate was dramatically reduced in the OP mice compared with the sham mice. At the same time, overexpression of miR-187 significantly attenuated the reduction of the relative bone repair rate in the OP mice $(P<0.05$, Fig. 2B). Also, the relative bone healing rate was notably decreased in the OP mice versus the sham mice. miR-187 overexpression could significantly reverse the decreased bone healing rate in the OP mice $(P<0.05$, Fig. $2 \mathrm{C})$. Moreover, we demonstrated that BARX2 expression was significantly elevated in the OP mice relative to the sham mice $(P<0.05$, Fig. 2D). This elevation could be weakened by miR-187 overexpression in the OP mice ( $P<0.001$, Fig. 2E). So, these findings manifested that miR-187 might have significantly promoted the reconstruction and healing of bone in the OP mice.

The miR-187 dramatically upregulated osteogenesis-related genes in hMSCs.

Subsequently, we investigated the impact of miR-187 on osteoblastic differentiation in hMSCs. We successfully isolated hMSCs from OP patients and then transfected the extracted hMSCs with miR-187 mimics or miR-187 inhibitor. qRT-PCR assay was conducted to evaluate the transfection efficiencies of miR-187 mimics and miR-187 inhibitor in hMSCs. As displayed in Fig. 3A, relative to their respective controls, miR-187 expression was markedly increased in the mimics group, and significantly decreased in the inhibitor group $(P<0.001)$. We confirmed that the osteogenic markers $(O C N, O P N, R U N X 2, B S P$, and ALP) were upregulated in the miR-187 mimics group, and dramatically downregulated in the miR-187 inhibitor group versus the NC group (Fig. 3B). The results of ALP staining showed that miR-187 mimics enhanced ALP activity, while miR-187 inhibitors weakened ALP activity in hMSCs $(P<0.01, P<0.001$, Fig. 3C). Next, we applied Alizarin Red staining to examine the influence of miR-187 on bone mineralization in hMSCs. As presented in Fig. 3D, the calcium nodules were significantly higher in the 
miR-187 mimics group than in the NC mimics group, and the calcium nodules were also significantly lower in the miR-187 inhibitor group than in the NC inhibitor group. These results implied that miR-187 facilitated osteoblast differentiation in hMSCs.

\section{BARX2 was a target of miR-187.}

Next, the mechanism by which miR-187 induces osteogenic differentiation was further explored. After experimental exploration, we discovered that BARX2 expression was significantly reduced at 14 and 28 days after induction of osteogenic differentiation in hMSCs $(P<0.01, P<0.001$, respectively, Fig. 4A and $4 B)$. We applied bioinformatics analysis to predict the possible binding sites between BARX2 and miR187, which are shown in Fig. 4C.

The luciferase reporter assay was conducted to identify the regulation between BARX2 and miR-187. The luciferase intensity was significantly weakened in HEK293 cells after co-transfection with wild-type BARX2 and miR-187 mimics ( $P<0.01$, Fig. 4D). We also confirmed that miR-187 mimics significantly downregulated BARX2 while miR-187 inhibitor significantly upregulated BARX2 in hMSCs $(P<0.01, P<$ 0.001 , Fig. $4 \mathrm{E})$. Overall, we have demonstrated that miR-187 significantly downregulated BARX2 through targeted regulation.

\section{Inhibition of miR-187 dramatically suppressed osteoblastic differentiation by BARX2.}

We further determined whether the pro-osteogenic effect of miR-187 on hMSCs was mediated by BARX2. hMSCs were individually transfected or co-transfected with miR-187 inhibitor or BARX2 siRNA. First, our qRT-PCR data displayed that miR-187 inhibitor significantly elevated the level of BARX2, while BARX2 expression was markedly reduced after BARX2 siRNA in miR-187 inhibitor-mediated hMSCs $(P<0.001$, Fig. 5A and 5B). Second, the results of the Western blot also exhibited that miR-187 inhibitor downregulated the osteogenic markers (OCN, OPN, RUNX2, BSP, and ALP) in hMSCs. These downregulations, which were mediated by miR-187 inhibitor, could also be significantly reversed by BARX2 siRNA in hMSCs (Fig. 5B). Next, ALP staining uncovered that miR-187 inhibitor notably lowered the ALP activity, and the ALP activity, which was inhibited by miR-187 inhibitor, could be elevated by BARX2 knockdown in hMSCs $(P<0.01, P<0.001$, Fig. $5 \mathrm{C})$. Alizarin Red staining results showed that the matrix mineralization state of hMSCs was reduced in the miR-187 inhibitor group versus the NC inhibitor group, and knockdown of BARX2 enhanced the matrix mineralization of hMSCs, which was suppressed by the miR-187 inhibitor (Fig. 5D). Therefore, we have shown that the effect of miR-187 on osteogenic differentiation was achieved by regulating BARX2 in hMSCs.

\section{Discussion}

$\mathrm{OP}$ is a frequent systemic degenerative disease of the skeletal system [30]. Osteoporosis not only increases the risk of fracture but also affects the stability of fracture fixation and the healing process. Currently, osteoporosis has many pathogenic factors and a complicated pathogenesis [31]. There is no specific drug to treat osteoporosis clinically. The application of animal models is a vital link in the study 
of the etiology, pathology, therapy, and prognosis of human osteoporosis [32]. Postmenopausal osteoporosis is the most familiar type of osteoporosis, accounting for $80 \%$ of the total number of patients [2]. Ovariectomized female mice have become the most commonly applied animal model of osteoporosis. Its advantages include a single modeling factor, high success rate, good repeatability, and high reliability [33]. In our study, we also established the osteoporosis mouse model through bilateral ovariectomy.

The hMSCs are pluripotent stem cells with self-renewal capacity, and they can differentiate into osteoblasts under appropriate culture conditions [34]. Multiple studies have manifested that hMSCs have crucial clinical application value in promoting bone healing and bone regeneration $[35,36]$. Therefore, studies on the osteogenic differentiation mechanism of hMSCs contribute to the clinical application of hMSCs in osteoporosis. In our study, we also successfully isolated hMSCs from OP patients.

Increasing evidence has suggested that miRNAs with the potential to regulate the 3囚-UTR of specific genes might be of great importance in the osteogenic differentiation of hMSCs [36-39]. For instance, miR-128 was confirmed to suppress the osteogenic differentiation of hMSCs by the VEGF pathway [38]; miR-7-5p could notably accelerate the osteogenic differentiation of hMSCs through CMKLR1 [39]; miR224 was verified to induce osteoblastic differentiation of hMSCs by Rac1 [36]. Our current research revealed that miR-187 was significantly underexpressed in the femoral bones from OP mice and the serum of OP patients. These results are consistent with previous research in which miR-187 was significantly downregulated in osteoporotic fractures [40]. Meanwhile, we first disclosed that miR-187 could dramatically accelerate bone healing and bone repair in the OP mouse model and induce osteogenic differentiation of hMSCs. Currently, miR-187 has also been shown to have a significant moderating effect on disease progression, especially cancer [26, 41-44]. These results suggested that miR-187 had a significant contribution to the disease process.

BARX2, as a homeodomain factor of the Bar family, has been confirmed to function in cell adhesion, cellular differentiation, and cytoskeletal remodeling $[45,46]$. Recent research has suggested that BARX2 is closely associated with multiple diseases, especially solid tumors [47-49]. Another study has also reported that BARX2 could be regulated by miR-187 in a targeted way in oral carcinoma [50]. In our study, we uncovered that with the BARX2 gene as a target, miR-187 could inhibit BARX2. Our data has also shown that BARX2 knockdown could reverse the effect on OP, which was brought about by the miR-187 inhibitor, suggesting that the miR-187 inhibitor prevented osteoblast differentiation in hMSCs by targeting BARX2.

\section{Conclusions}

In summary, we proved that miR-187 could markedly facilitate bone reconstruction and healing in a mouse model of OP in vivo and induce osteoblastic differentiation of hMSCs through BARX2 in vitro. Therefore, our results provide possible new targets for the treatment of osteoporotic fractures. They also 
provide novel evidence for further exploration of the interactions between miR-187 and BARX2 during osteogenic differentiation.

\section{Abbreviations}

$\mathrm{OP}$

Osteoporosis; hMSCs:Human marrow-derived mesenchymal stem cells; miRNAs:MicroRNAs;

BARX2:BarH-like homeobox 2; SPF:specific pathogens free; NC:Negative control; a-MEM:a-modified Eagle's medium; FBS:fetal bovine serum; BMD:bone mineral density; qRT-PCR:Quantitative real-time

\section{Declarations}

\section{Ethics approval and consent to participate}

All protocols followed the requirements of the Animal Experiment Center of the Institute of Radiation Medicine of the Chinese Academy of Medical Sciences. Informed consent was provided from all patients. This study was allowed by the ethics committee of the The First Affiliated Hospital of Jinan University.

\section{Consent for publication}

Not applicable.

\section{Availability of data and materials}

The data used to support the findings of this study are available from the corresponding author upon request.

\section{Competing interests}

The authors declare no competing interests.

\section{Funding}

This research did not receive any specific grant from funding agencies in the public, commercial, or notfor-profit sectors.

\section{Authors' contributions}

JZ, FP, and ZGZ were responsible for the research design. BST and JL contributed to various aspects of data acquisition, analysis, and interpretation. JZ and ZGZ drafted the paper. All authors critically revised the paper. All authors read and approved the final manuscript.

\section{Acknowledgments}

Not applicable. 


\section{References}

1. Ensrud KE, Crandall CJ. Osteoporosis. Ann Intern Med. 2017;167(3):Itc17-32.

2. Black DM, Rosen CJ. Clinical Practice. Postmenopausal Osteoporosis. N Engl J Med. 2016;374(3):254-62.

3. Aspray TJ, Hill TR. Osteoporosis and the Ageing Skeleton. Subcell Biochem. 2019;91:453-76.

4. Kerschan-Schindl K. Prevention and rehabilitation of osteoporosis. Wien Med Wochenschr. 2016;166(1-2):22-7.

5. Curtis EM, Moon RJ, Harvey NC, Cooper C. The impact of fragility fracture and approaches to osteoporosis risk assessment worldwide. Bone. 2017;104:29-38.

6. Anagnostis P, Gkekas NK, Potoupnis M, Kenanidis E, Tsiridis E, Goulis DG. New therapeutic targets for osteoporosis. Maturitas. 2019;120:1-6.

7. Yoon BH, Lee JK, Choi DS, Han SH. Prevalence and Associated Risk Factors of Sarcopenia in Female Patients with Osteoporotic Fracture. Journal of Bone Metabolism. 2018;25(1).

8. Yoshizawa T, Nishino T, Okubo I, Yamazaki M. Cost-effectiveness analysis of drugs for osteoporosis treatment in elderly Japanese women at high risk of fragility fractures: comparison of denosumab and weekly alendronate. Arch Osteoporos. 2018;13(1):94.

9. Fleurence RL, Iglesias CP, Johnson JM. The cost effectiveness of bisphosphonates for the prevention and treatment of osteoporosis: a structured review of the literature. Pharmacoeconomics. 2007;25(11):913-33.

10. Khosla S, Hofbauer LC. Osteoporosis treatment: recent developments and ongoing challenges. Lancet Diabetes Endocrinol. 2017;5(11):898-907.

11. Bandeira L, Lewiecki EM, Bilezikian JP. Romosozumab for the treatment of osteoporosis. Expert Opin Biol Ther. 2017;17(2):255-63.

12. Park HC, Son YB, Lee SL, Rho GJ, Kang YH, Park BW, et al. Effects of Osteogenic-Conditioned Medium from Human Periosteum-Derived Cells on Osteoclast Differentiation. Int J Med Sci. 2017;14(13):1389-401.

13. Kim JH, Kim M, Jung HS, Sohn Y. Leonurus sibiricus L. ethanol extract promotes osteoblast differentiation and inhibits osteoclast formation. Int J Mol Med. 2019;44(3):913-26.

14. Chen Q, Shou P, Zheng C, Jiang M, Cao G, Yang Q, et al. Fate decision of mesenchymal stem cells: adipocytes or osteoblasts? Cell Death Differ. 2016;23(7):1128-39.

15. Wang C, Meng H, Wang X, Zhao C, Peng J, Wang Y. Differentiation of Bone Marrow Mesenchymal Stem Cells in Osteoblasts and Adipocytes and its Role in Treatment of Osteoporosis. Med Sci Monit. 2016;22:226-33.

16. Sølling ASK, Harsløf T, Langdahl B. Current Status of Bone-Forming Therapies for the Management of Osteoporosis. Drugs Aging. 2019;36(7):625-38. 
17. Rachner TD, Hofbauer LC, Göbel A, Tsourdi E. Novel therapies in osteoporosis: PTH-related peptide analogs and inhibitors of sclerostin. J Mol Endocrinol. 2019;62(2):R145-r54.

18. Cui J, Zhou B, Ross SA, Zempleni J. Nutrition, microRNAs, and Human Health. Adv Nutr. 2017;8(1):105-12.

19. Li Y, Liu R, Li J, Dong X, Shen J, Wu S. Isoflurane promotes proliferation and invasion of cervical carcinoma cells via downregulation of miR-375 expression. Eur J Gynaecol Oncol. 2019;40(3):36872.

20. Vienberg S, Geiger J, Madsen S, Dalgaard LT. MicroRNAs in metabolism. Acta Physiol (Oxf). 2017;219(2):346-61.

21. Feng $Q$, Zheng $S$, Zheng J. The emerging role of microRNAs in bone remodeling and its therapeutic implications for osteoporosis. Biosci Rep. 2018;38(3).

22. Zhao W, Shen G, Ren H, Liang D, Yu X, Zhang Z, et al. Therapeutic potential of microRNAs in osteoporosis function by regulating the biology of cells related to bone homeostasis. J Cell Physiol. 2018;233(12):9191-208.

23. Zhang QL, Wang W, Alatantuya, Dongmei, Lu ZJ, Li LL, et al. Down-regulated miR-187 promotes oxidative stress-induced retinal cell apoptosis through P2 $\times 7$ receptor. Int $\mathrm{J}$ Biol Macromol. 2018;120(Pt A):801-10.

24. Liu CJ, Lin JS, Cheng HW, Hsu YH, Cheng CY, Lin SC. Plasma miR-187* is a potential biomarker for oral carcinoma. Clin Oral Investig. 2017;21(4):1131-8.

25. Zhu QL, Li Z, Lv CM, Wang W. MiR-187 influences cisplatin-resistance of gastric cancer cells through regulating the TGF- $\beta /$ Smad signaling pathway. Eur Rev Med Pharmacol Sci. 2019;23(22):9907-14.

26. Gulinaer AJ, Ju AN, Gao M, Luo Y, Bo YL. Over-expression of miR-187 inhibited cell proliferation and metastasis of glioma via down-regulating SMAD1. Eur Rev Med Pharmacol Sci. 2019;23(24):1090817.

27. Mulrane L, Madden SF, Brennan DJ, Gremel G, McGee SF, McNally S, et al. miR-187 is an independent prognostic factor in breast cancer and confers increased invasive potential in vitro. Clin Cancer Res. 2012;18(24):6702-13.

28. Tang L, He S, Zhu Y, Feng B, Su Z, Liu B, et al. Downregulated miR-187 contributes to the keratinocytes hyperproliferation in psoriasis. J Cell Physiol. 2019;234(4):3661-74.

29. Garmilla-Ezquerra P, Sañudo C, Delgado-Calle J, Pérez-Nuñez MI, Sumillera M, Riancho JA. Analysis of the bone microRNome in osteoporotic fractures. Calcif Tissue Int. 2015;96(1):30-7.

30. Coughlan T, Dockery F. Osteoporosis and fracture risk in older people. Clin Med (Lond). 2014;14(2):187-91.

31. Armas LA, Recker RR. Pathophysiology of osteoporosis: new mechanistic insights. Endocrinol Metab Clin North Am. 2012;41(3):475-86.

32. Komori T. Animal models for osteoporosis. Eur J Pharmacol. 2015;759:287-94. 
33. Zhang Z, Ren H, Shen G, Qiu T, Liang, Yang Z, et al. Animal models for glucocorticoid-induced postmenopausal osteoporosis: An updated review. Biomed Pharmacother. 2016;84:438-46.

34. Medhat D, Rodriguez $\mathrm{Cl}$, Infante A. Immunomodulatory Effects of MSCs in Bone Healing. Int J Mol Sci. 2019;20(21).

35. Costa V, Carina V, Raimondi L, De Luca A, Bellavia D, Conigliaro A, et al. MiR-33a Controls hMSCS Osteoblast Commitment Modulating the Yap/Taz Expression Through EGFR Signaling Regulation. Cells. 2019;8(12).

36. Cai Q, Zheng P, Ma F, Zhang H, Li Z, Fu Q, et al. MicroRNA-224 enhances the osteoblastic differentiation of hMSCs via Rac1. Cell Biochem Funct. 2019;37(2):62-71.

37. Hodges WM, O'Brien F, Fulzele S, Hamrick MW. Function of microRNAs in the Osteogenic Differentiation and Therapeutic Application of Adipose-Derived Stem Cells (ASCs). Int J Mol Sci. 2017;18(12).

38. Zhang W, Yao C, Wei Z, Dong Q. miR-128 promoted adipogenic differentiation and inhibited osteogenic differentiation of human mesenchymal stem cells by suppression of VEGF pathway. $J$ Recept Signal Transduct Res. 2017;37(3):217-23.

39. Chen B, Meng J, Zeng YT, Du YX, Zhang J, Si YM, et al. MicroRNA-7-5p regulates osteogenic differentiation of hMSCs via targeting CMKLR1. Eur Rev Med Pharmacol Sci. 2018;22(22):7826-31.

40. Garmilla-Ezquerra P, Sanudo C, Delgado-Calle J, Perez-Nunez MI, Sumillera M, Riancho JA. Analysis of the bone microRNome in osteoporotic fractures. Calcif Tissue Int. 2015;96(1):30-7.

41. Zhu QL, Li Z, Lv CM, Wang W. MiR-187 influences cisplatin-resistance of gastric cancer cells through regulating the TGF-beta/Smad signaling pathway. Eur Rev Med Pharmacol Sci. 2019;23(22):990714.

42. Yue J, Si Y, Zhu T, Yang J, Xu X, Fang Y, et al. MicroRNA-187 Reduces Acute Ischemic Renal Podocyte Injury via Targeting Acetylcholinesterase. J Surg Res. 2019;244:302-11.

43. Liang H, Luo R, Chen X, Zhao Y, Tan A. miR-187 inhibits the growth of cervical cancer cells by targeting FGF9. Oncol Rep. 2017;38(4):1977-84.

44. Cui C, Shi X. miR-187 inhibits tumor growth and invasion by directly targeting MAPK12 in osteosarcoma. Exp Ther Med. 2017;14(2):1045-50.

45. Meech R, Edelman DB, Jones FS, Makarenkova HP. The homeobox transcription factor Barx2 regulates chondrogenesis during limb development. Development. 2005;132(9):2135-46.

46. Stevens TA, lacovoni JS, Edelman DB, Meech R. Identification of novel binding elements and gene targets for the homeodomain protein BARX2. J Biol Chem. 2004;279(15):14520-30.

47. Yang F, Shao C, Wei K, Jing X, Qin Z, Shi Y, et al. miR-942 promotes tumor migration, invasion, and angiogenesis by regulating EMT via BARX2 in non-small-cell lung cancer. J Cell Physiol. 2019;234(12):23596-607.

48. Mi Y, Zhao S, Zhang W, Zhang D, Weng J, Huang K, et al. Down-regulation of Barx2 predicts poor survival in colorectal cancer. Biochem Biophys Res Commun. 2016;478(1):67-73. 
49. Zhang Y, Zhang JX, Huang LL, He LJ, Liao YJ, Lai YR, et al. Low expression of BARX2 in human primary hepatocellular carcinoma correlates with metastasis and predicts poor prognosis. Hepatol Res. 2015;45(2):228-37.

50. Lin SC, Kao SY, Chang JC, Liu YC, Yu EH, Tseng SH, et al. Up-regulation of miR-187 modulates the advances of oral carcinoma by targeting BARX2 tumor suppressor. Oncotarget. 2016;7(38):6135565 .

\section{Figures}

A

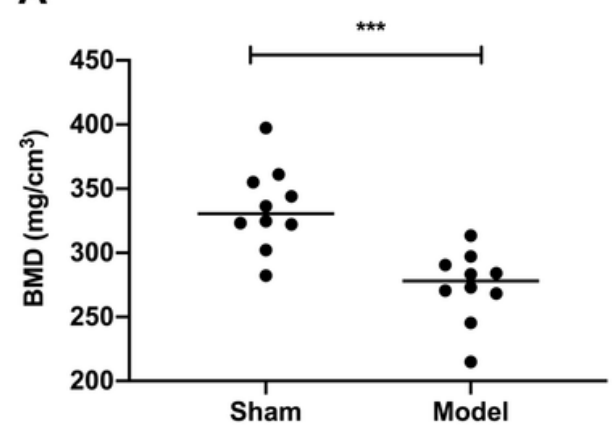

B

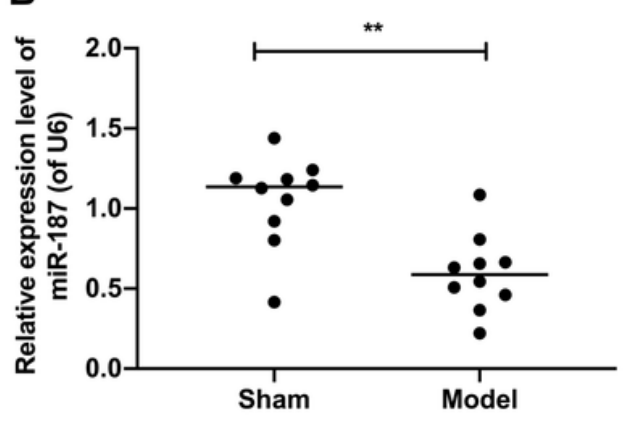

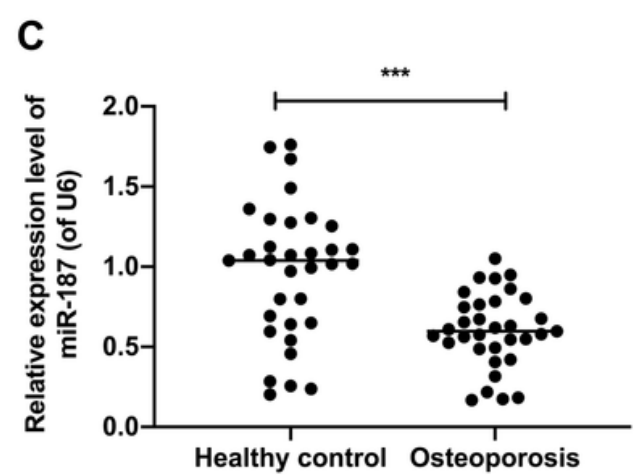

\section{Figure 1}

The miR-187 was significantly downregulated in osteoporosis (OP). The OP mouse model was established through bilateral ovariectomy. (A) The BMD value was examined using the iDXA in the left femur of the sham group and the OP model group. (B) miR-187 expression was identified through qRTPCR assay in the femoral bone of the sham and model groups. (C) qRT-PCR assay was conducted to determine the expression of miR-187 in the serum of healthy controls $(n=33)$ and OP patients $(n=33)$. $\star \star P<0.01, * \star * P<0.001$. 
A

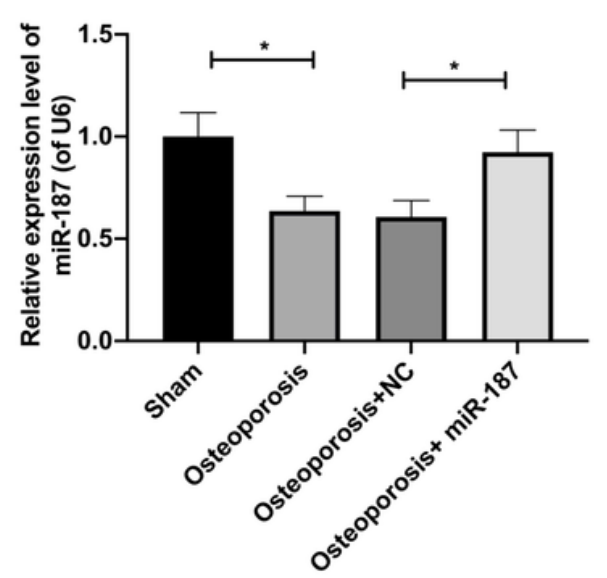

\section{D}

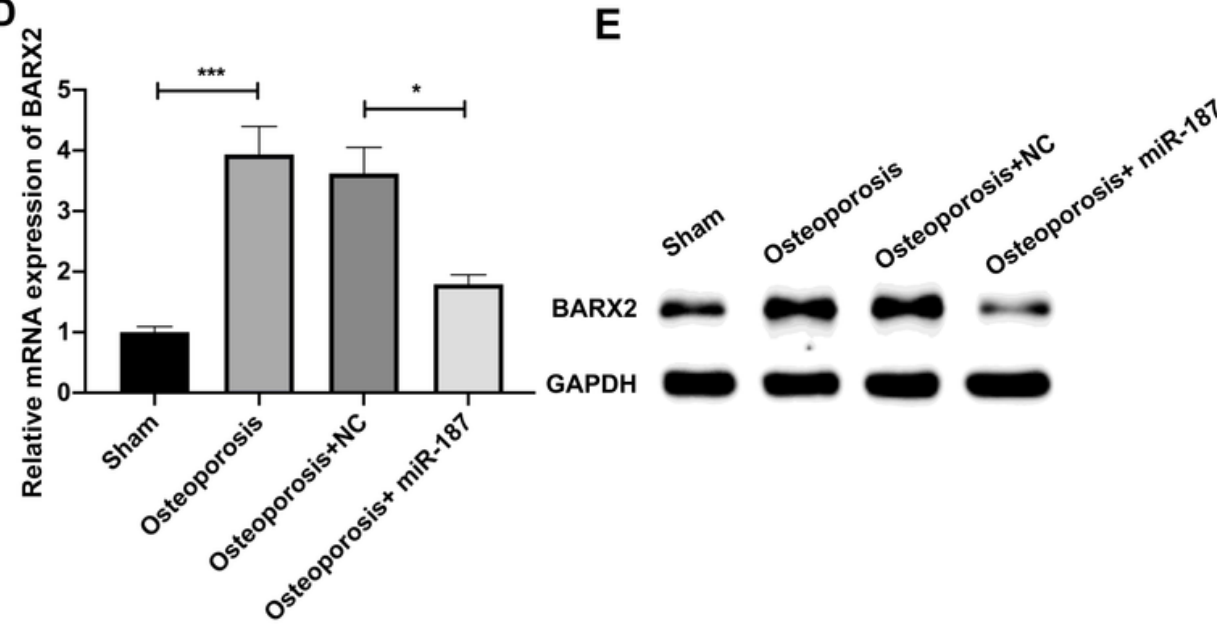

C
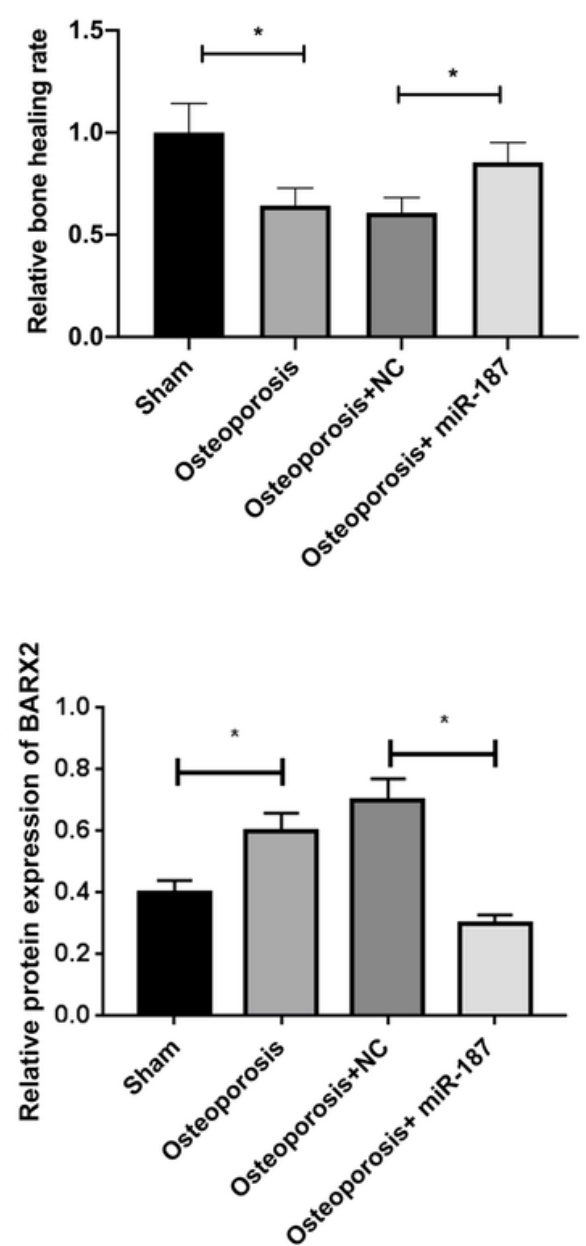

Figure 2

The miR-187 markedly promoted the reconstruction and healing of bone and downregulated BARX2. The OP mice were injected with NC or miR-187 lentivirus through the tail vein. (A) qRT-PCR analysis was conducted of miR-187 expression in the femoral bone of mice. (B) Bone repair in each group was assessed by toluidine blue staining. (C) Bone healing was determined and counted through $X$-ray. (D-E) BARX2 expression was confirmed by qRT-PCR and western blot assays. ${ }^{*} P<0.05$, $* \star * P<0.001$. 


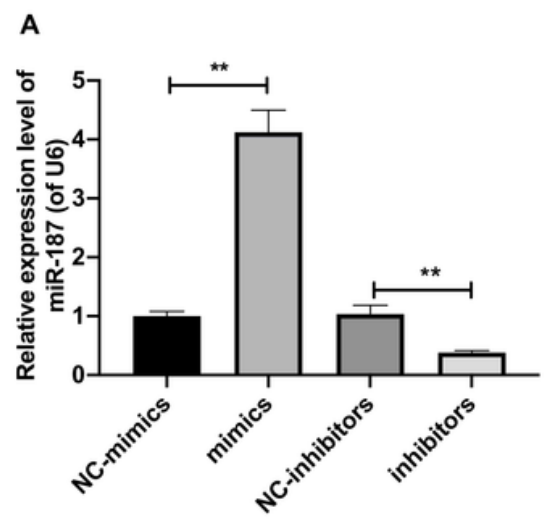

C NC-mimics

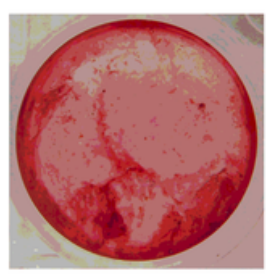

mimics

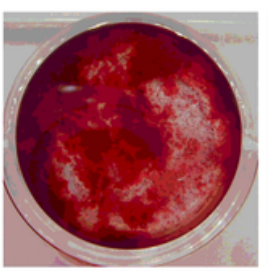

B
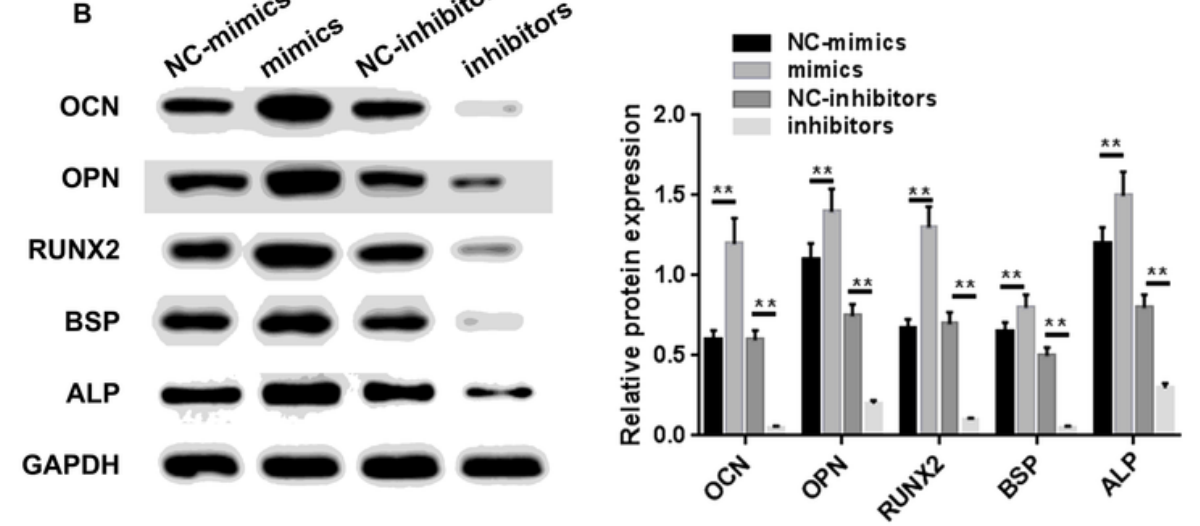

D

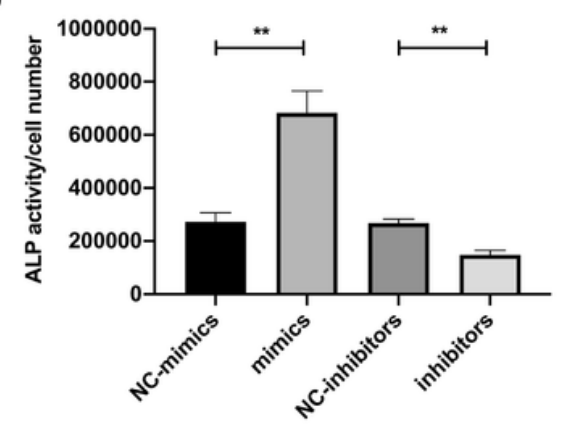

Figure 3

The miR-187 upregulated osteogenesis-related genes in hMSCs. hMSCs were extracted from OP patients, and hMSCs were transfected with miR-187 mimics or miR-187 inhibitor. (A) Expression of miR-187 was evaluated using the qRT-PCR assay. (B) The expressions of OCN, OPN, RUNX2, BSP, and ALP in transfected hMSCs were verified using Western blot assays. (C) The evaluation of ALP activity through ALP staining in hMSCs transfected with NC, miR-187 mimics or miR-187 inhibitor. (D) The matrix mineralization state of hMSCs was evaluated by alizarin red staining. ${ }^{\star *} \mathrm{P}<0.01,{ }^{* \star *} \mathrm{P}<0.001$. 
A

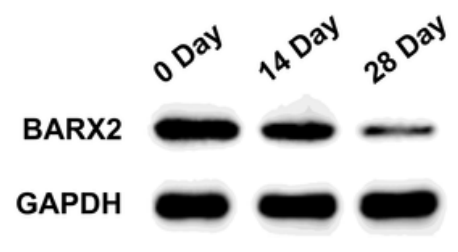

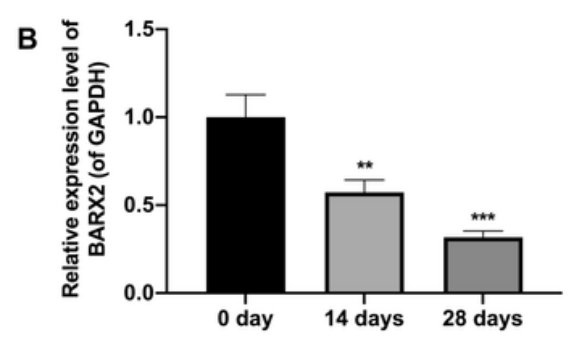

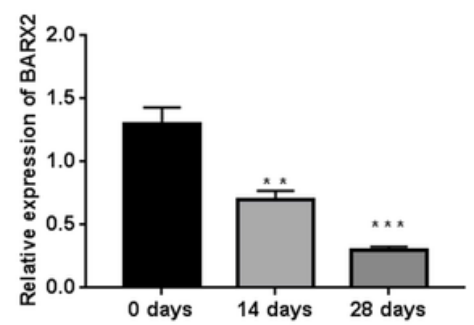

C
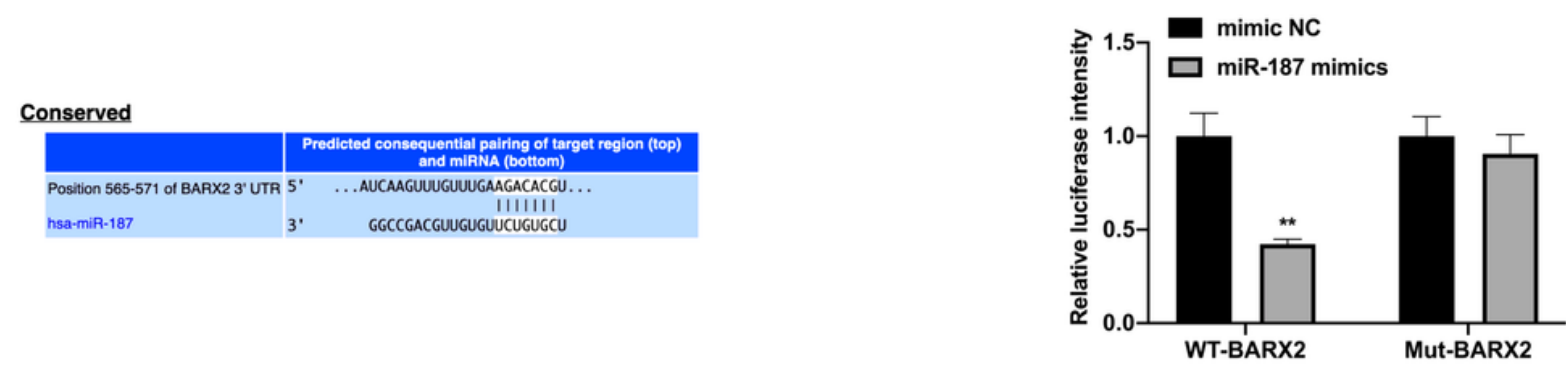

D

E

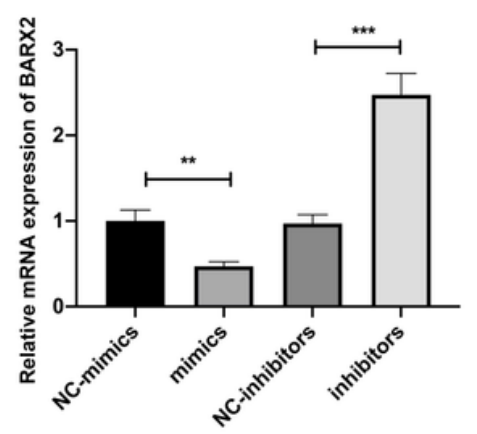

BARX2
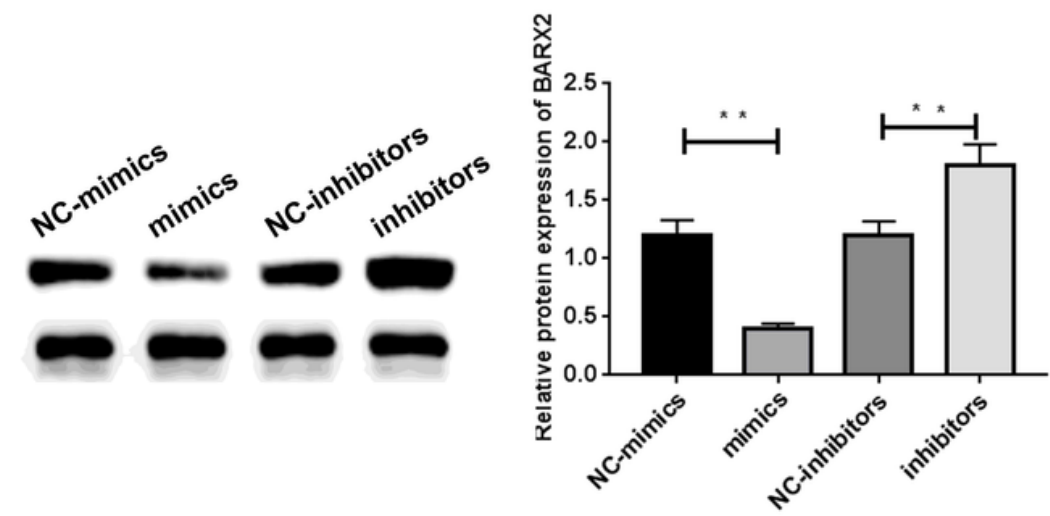

Figure 4

BARX2 was a target of miR-187. (A-B) qRT-PCR and western blot assays were conducted to examine BARX2 expression in hMSCs after induction for 0, 14, and 28 days. (C) The sequence of miR-187 matched the 3'-UTR of BARX2 as exhibited through bioinformatics analysis. (D) The 293T cells were cotransfected with miR-187 mimics or mimic NC and the luciferase constructs carrying BARX2, and the fluorescence intensity was monitored using a luciferase assay. (E) The level of BARX2 was confirmed using qRT-PCR and Western blot assays in hMSCs after transfection with miR-187 mimics or miR-187 inhibitor. ${ }^{* *} \mathrm{P}<0.01,{ }^{*} * * \mathrm{P}<0.001$. 
A

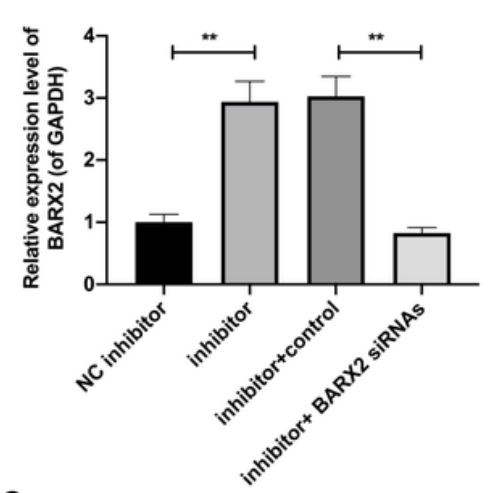

C

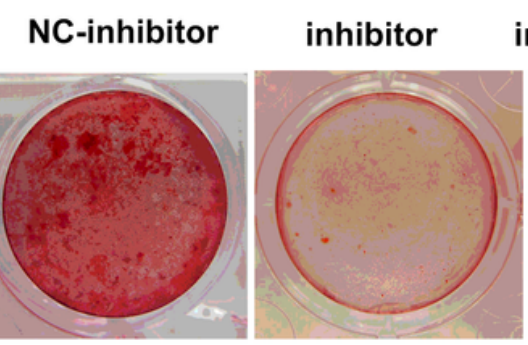

B

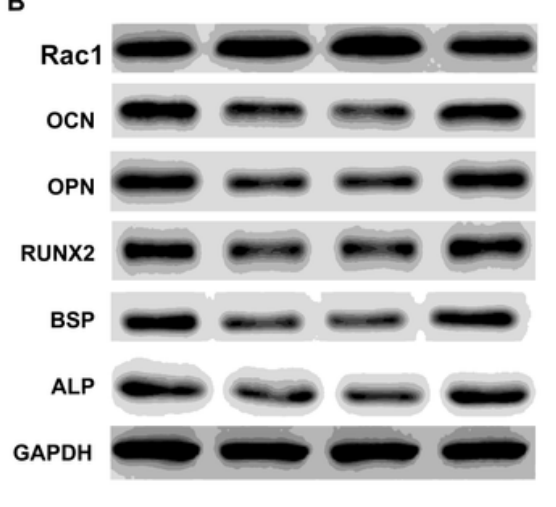

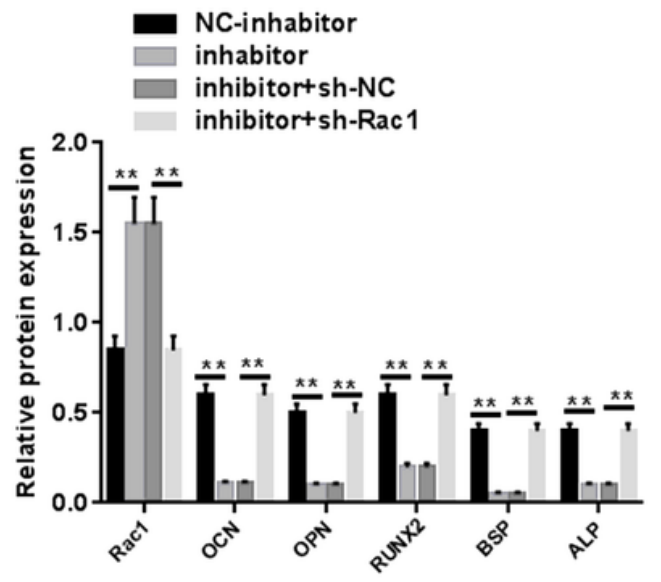

D

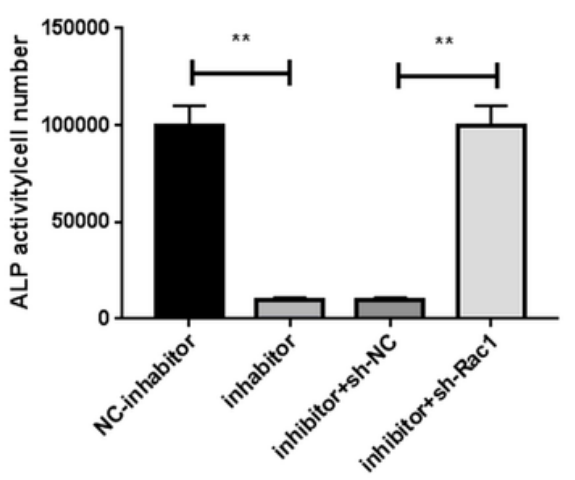

Figure 5

Inhibition of miR-187 suppressed osteoblastic differentiation by BARX2. miR-187 inhibitor or BARX2 siRNA were individually transfected or co-transfected into hMSCs. (A) qRT-PCR was conducted to determine the mRNA level of BARX2 in each group of hMSCs. (B) The levels of BARX2, OCN, OPN, RUNX2, BSP, and ALP were confirmed in the transfected hMSCs. (C) ALP staining was adopted to assess the impact of miR-187 inhibitor and BARX2 knockdown on ALP activity in each group of hMSCs. (D) Alizarin Red staining was utilized to confirm the changes in the matrix mineralization state of hMSCs in each group. ${ }^{\star *} \mathrm{P}<0.01,{ }^{*} * \mathrm{P}<0.001$.

\section{Supplementary Files}

This is a list of supplementary files associated with this preprint. Click to download.

- WB.pdf

- NC3RsARRIVEGuidelinesChecklistfillable.pdf 